\title{
Gravitation of Blockchain in Shared Services: The Next Phase of Service Delivery Strategy
}

\author{
Vipin K. Suri ${ }^{1}$, Marianne D. Elia ${ }^{1}$, and Jos van Hillegersberg ${ }^{2(\bowtie)}$ \\ ${ }^{1}$ Shared Services International Inc, Mississauga, Canada \\ 2 Faculty of Behavioral, Management and Social Sciences, Industrial Engineering and Business \\ Information Systems, University of Twente, P.O. Box 217, 7500 AE Enschede, The Netherlands \\ j.vanhillegersberg@utwente.nl
}

\begin{abstract}
A Blockchain is an immutable, tamper-proof, shared ledger of state changes of a digital asset. It is an incorruptible digital ledger of economic transactions that can be programmed to record not just financial transactions but virtually everything of value. This digital ledger is managed via a distributed network across many nodes that can verify and confirm those transactions through consensus. The implications of the technology are far-reaching but there are conditions that should be met in order for Blockchain to be a viable solution. The purposes of this research are to (1) explore the current Blockchain use cases in Shared Services (2) understand the value created by Blockchain in Supply Chain Management and (3) study the tactical challenges in adopting a Blockchain strategy in Shared Services. In addition to a literature review conducted, we conducted in-depth interviews with selected Shared Services Leaders and experts. Results of our research indicate that Blockchain technology can deliver on expectations and implementation in Shared Services organizations will require simple steps. This study provides the data necessary for executives to build a business case for applying Blockchain technology in Shared Services and investigates the potential that Blockchain has to revolutionize industry and deliver gains in speed, security, transparency, traceability and accountability for a wide range of business processes.
\end{abstract}

Keywords: Blockchain · Data integrity $\cdot$ Hashing $\cdot$ Block $\cdot$ Encryption ·

Distributed ledger · Market maker · Industry group · Regulator · Node selection ·

Digital asset $\cdot$ Intermediaries

\section{Introduction}

Blockchain creates a ledger of all transactions in a given process. Each ledger entry is autonomous so can provide insights into cash flow and add extra security in exchanges involving cryptocurrency. Virtually incorruptible due to the fact that the data contained within a ledger entry cannot be copied, Blockchain is the backbone of cryptocurrency transactions. It can track any item of value, so is particularly useful in shipping as well as online retail, making it a powerful tool for business units with the vision to utilize this tool. 
A Blockchain facilitates secure online transactions. It is a decentralized and distributed digital ledger that is used to record transactions across many computers so that the record cannot be altered retroactively without the alteration of all subsequent blocks and the collusion of the network. This allows the participants to verify and audit transactions. While the knowledge on Blockchain technology and applications is rapidly expanding, there is little insight on how Blockchain can enhance delivery of Shared Services.

Using a combination of available literature study and in-depth interviews with experts and practitioners we address the following research questions:

1. What are the key factors that facilitate adoption of Blockchain technology in Shared Services?

2. What are the challenges in adopting Blockchain technology in Shared Services?

3. What processes are ideal candidates for Blockchain technology?

4. What is the promise of Blockchain technology in Shared Services?

To develop a common understanding of the terms used in Blockchain technology, we offer the following definitions:

Hashing - Hashing is a form of cryptographic security.

Encryption - Encryption is a two-step process used to first encrypt and then decrypt a message, hashing condenses a message into an irreversible fixed-length value, or hash. Public Key - A public identifier that can be freely shared with others; this is the identity on the Blockchain

Private Key - A key that must never be shared with anyone

This paper is organized in following sections: Sect. 1 has just provided an introduction to Blockchain technology and has set the stage for our study. Section 2 provides a summary of our literature review related to Blockchain technology and use cases in Shared Services for Blockchain. Section 3 presents our data collection methods. Section 4 provides findings and analysis of data collected and Sect. 5 outlines the conclusions and future research.

\section{Literature Review}

There is great confusion and debate about what a blockchain even is - some people argue it has become a meaningless buzzword - but the standard definition describes a shared, decentralized, cryptographically secure, immutable digital ledger. In the broadest terms, a blockchain allows a group of strangers to agree on a state-of-affairs and to proceed together on the basis of that covenant. Bitcoin's blockchain is meant to supplant the powerful middlemen called banks, but in theory a blockchain could replace any kind of institution - a credit agency, a social media service - that exists to safeguard a changing set of historical records. We pay these centralized entities handsomely for their custodial services, not only in the form of the rents they charge but in the control they exert over our lives. The blockchain, in theory, afford us new opportunities to solve complex 
coordination problems without letting the incumbent coordinators extract so much value in the process. This had, of course, been the initial premise of the internet itself. Its great collaborative potential, however, had been funneled into the leviathans of Amazon, Facebook, and Google - a new and massively powerful set of trusted third parties. The blockchain pointed the way to the sunlit uplands of a genuinely decentralized world. A loose culture of entrepreneurs and cyberpunks came together in what felt like a special moment of experimental ferment. Most of these early blockchain innovators just took the original cryptocurrency's source code, made their preferred changes, and launched their alternative versions as distinct cryptocurrencies, it was as if they'd modified the DNA of an existing species to create a new, reproductively isolated branch of the family tree [1].

Over the past couple of years, the emergence of Robotic Process Automation (RPA) tools ranging from desktop 'fixes' to server based, end-to-end driven process automation have been a major contributor towards real-time, digital, automated operations. However, flying somewhat under the radar, a novel business-to-business transaction network has been promising to fuel the next step change in the digital revolution. It's called blockchain and is defined as a 'peer-to-peer distributed ledger forged by consensus, combined with a system for 'smart contracts' and other assistive technologies, which together can be used to build a new generation of transactional applications that establishes trust, accountability and transparency at their core, while streamlining business processes and legal constraints.

Blockchain gets its name from blocks, a series of transactions (i.e. a bank statement); and a chain containing a sequential set of transaction records (i.e. blocks), in the order in which they occurred. The beauty of blockchain technology is that it works across a network and allows for instant, seamless business-to-business transactions based on preagreed and confirmed data nodes - effectively, 'an operating system for marketplaces, data-sharing networks, micro-currencies and decentralized digital communities with the potential to vastly reduce the cost and complexity of getting things done in the real world'. Blockchain's ability to track ownership without involving third-parties opens the door to decentralized transactions in a way that positively disrupts current business models.

Consider the many and various interactions a business has with the external markets: a key hurdle to transactions is the invariable slowdown that results from separate parties awaiting clearance for prescribed activities. In the world of blockchain, these kinds of interactions are pre-approved and take place instantly across networks based on Distributed Ledger Technology, which stores all the information needed to greenlight transactions in real-time.

To date, the financial services sector has been the most active - clearing houses, exchanges and the like have large volume, multiple transactions. Many have proof of concepts and pilots in quite advanced stages and some are already working with production systems and data. Within the Shared Services space, blockchain offers interesting opportunities for the P2P and Supply Chain process. E-invoicing, for example, could overcome some of its challenges, particularly with larger suppliers, as the technology removes the need for reconciliation. Supply chain is a natural fit, as there are lots of steps - electronic ordering, invoicing and acknowledgement - that can be broken down. 
A shared ledger between a customer and all its suppliers would support optimization [2].

Digitalization of the accounting system is still in its infancy compared to other industries, some of which have been massively disrupted by the advances of technology. Some of the reasons may be found in the exceptionally high regulatory requirements in respect to validity and integrity.

The entire accounting system is built, such that forgery is impossible or at least very costly. To achieve this, it relies on mutual control mechanisms, checks and balances. This inevitably affects every day's operations. Among other things there are systematic duplication of efforts, extensive documentations and periodical controls. Most of them are manual, labor intensive tasks and far from being automated. To date, that seemed to be the sacrifice of revealing the truth. The recently emerged Blockchain is a trustless, distributed ledger that is openly available and has negligible costs of use. The use of the Blockchain for accounting use-cases is hugely promising. From simplifying the compliance with regulatory requirements to enhancing the prevalent double entry bookkeeping, anything is imaginable. Modern financial accounting is based on a double entry system. Double entry bookkeeping revolutionized the field of financial accounting during the Renaissance period; it solved the problem of managers knowing whether they could trust their own books. However, to gain the trust of outsiders, independent public auditors also verify the company's financial information. Each audit is a costly exercise, binding the company's accountants for long time periods. Stakeholders place their trust in the auditors retained by management to vouch for them. An obvious problem of agency is created by this arrangement (Fig. 1). Do auditors work for the managers who hire and pay them or for the public that relies on their integrity in order to make decisions? [3].

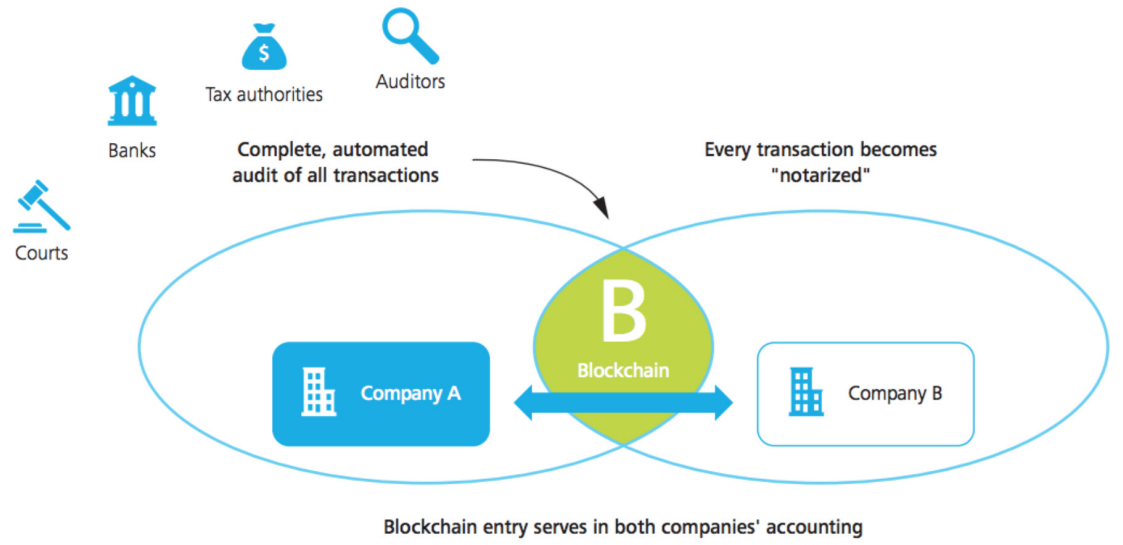

Fig. 1. Blockchain technology enables complete, conclusive verification without a trusted party [3]

By design, blockchains are inherently resistant to modification of any stored data. Functionally, a blockchain can serve as an open, distributed ledger that can record transactions between two parties efficiently and in a verifiable and permanent way. Blockchain 
can be used as a source of verification for reported transactions. An example might be where, instead of asking clients for bank statements or sending confirmation requests to third parties, auditors can easily verify the transactions on publically available blockchain ledgers. The automation of this verification process will drive cost efficiencies in the audit environment. The days of sample based substantive testing will soon be challenged, as auditors will resort to blockchain technology to test the whole population of transactions within the period under observation. This extensive coverage will drastically improve the level of assurance gained in affected audit engagements. In the Bitcoin blockchain, a transaction of low value currently takes approximately $10 \mathrm{~min}$ to be validated as a single block verification is deemed appropriate. The more blocks elapse before a transaction is considered as verified, i.e. the further in the chain, the more the related transactions are immutable. Typically, a high value transaction will take approximately $1 \mathrm{~h}$ to be verified (6 blocks). Contrast this with traditional financial transactions where information might take up to a month or more to be cleared. This pseudo real-time verification blockchain characteristic could also impact the audit process. Instead of assessments at year end (or interim), audit firms will be in a position to perform continuous on-line assessments throughout the period under audit [4].

The benefit of utilizing a blockchain in this manner is it would create a standardized record keeping process, which allows auditors to verify a large portion of the most important data behind the financial statements automatically. This would dramatically reduce the cost, labor and time necessary to conduct an audit, freeing up auditors to spend more time on areas where they can add value such as, working on very complex transactions or on internal control mechanisms (Fig. 2).
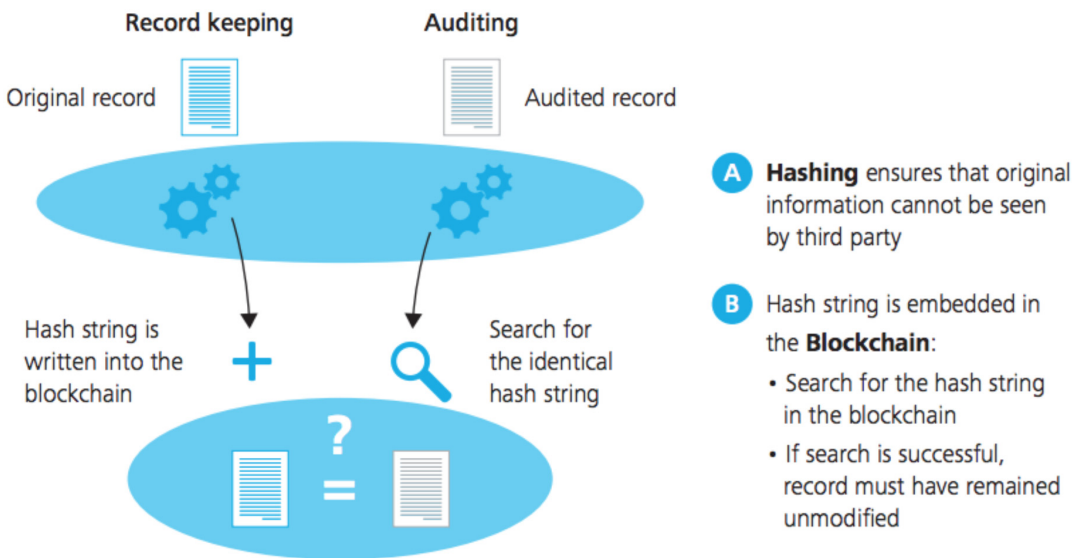

Fig. 2. One approach to verify the integrity of records using Blockchain [3].

There are many potential uses for HR that could benefit from blockchain technology. From a hiring perspective, access to academic credentials and certifications could reduce the amount of time recruiters spend verifying information. A wealth of information about potential candidates could be stored on blockchain making it easy for recruiters to find and verify everything from educational levels, postgraduate certifications and 
accomplishments. This would give a competitive edge to organizations that recruit based on verifiable credentials to find candidates that are overlooked by other organizations that only focus on traditional education and resumes. Once a credential is recorded to the blockchain, it can't be changed, deleted or faked. A fraudulent credential may look like an issued one, but it can be verified against the original blockchain record to confirm if it is valid or not. Digital credentials would remain secure, even if the company issuing them were to be hacked. Also, if schools or businesses shut down, a candidate's records would remain available. This means their records can be accessed no matter where in the world an individual decides to seek employment. The benefits for international employees are that a blockchain can process payroll faster and less expensively, avoiding international currency trade fees. By cutting out the intermediary, payments could come within hours rather than days - similar to cross-border payments [5].

Ireland-based global consulting firm Accenture and Italy-based insurance giant Generali Group's employee benefits unit have debuted a blockchain solution for employee benefits. The partnership was announced in a press release from the firms on April 16, 2019. Generali Employee Benefits (GEB) is an employee benefit solutions provider and global business line of Italy's largest insurance firm and 8th largest globally. The press release states that GEB operates the largest employee benefits solutions network worldwide. The blockchain solution will reportedly facilitate data sharing for parties involved in the reinsurance process for captive and pooling services. It will also reportedly improve procedures' reliability through the use of smart contracts and automated reconciliation. Captive insurance refers to underwriters who are wholly-owned by their insured clients, while reinsurance pooling services are a risk financing mechanism used by insurers to increase their ability to underwrite specific types of risks. The blockchain tool is designed to innovate and simplify GEB's existing employee benefits operating model for captive services, which spans policies such as life, disability, accident and healthcare insurance. The solution is reportedly supported by Generali and inspired by the work of the insurance industry collaborative blockchain initiative B3i. The solution's launch reportedly comes after the successful completion of a prototype in 2018, involving major agricultural firm Syngenta, as well as Spanish, Swiss and Serbian local insurers. The prototype was found to provide significant benefits for the employee benefits sector by establishing effective integration of systems, data and processes for all stakeholders, improving data quality and saving time and costs. GEB's CEO stated in the press release that in its provision of a seamless ecosystem, "blockchain will change not only our Network but the employee benefits industry as we know it". As reported, B3i Service AG - the Swiss blockchain startup founded by B3i in March of last year - expanded its group of investors this month as part of its ongoing funding round. The startup is focused on multi-company syndicated risk placement, accounting, and claims, and has developed a product based on R3 Corda's distributed ledger technology [6].

The advent of emerging digital technologies, such as robotic process automation (RPA), analytics, machine learning, cloud, and blockchain, is transforming the shared services landscape. Most large global businesses that use shared services models for a range of business functions, from finance and procurement to HR, are revamping the way they structure their support ecosystems. The convergence of a set of digital capabilities encourages organizations to abandon the individual or shared functional silos of the 
traditional shared services model. Instead, they are adopting a new integrated business services (IBS) model. When applied across functions such as finance, IT, HR, procurement, security, field operations, and others, it forms the basis of multi-functional integration. It has the power to transform business performance and become the gold standard of providing business services. Successful adoption ushers in savings through improved operational efficiency while also setting the stage for future growth and acquisition activity. The blockchain technology is transforming the way in which data is stored, verified, and accessed. Enabled by digital technology, a blockchain is a form of ledger that records data or transactions in a chronological order. The ledger is automatically replicated and maintained by each participant to create a decentralized, consensus controlled, tamperproof public ledger of assets and transactions. Blockchain is set to revolutionize finance and accounting. Unlike current financial transactions, blockchain does not rely on any single party, middleman, or regulatory intermediary. Consensus authentication drives blockchain as the record of transactions is shared among all parties. The transaction becomes more secure and transparent. This transformation will be far reaching. According to Outlier Ventures, around 250 major corporations are active in blockchain, either through in-house developments or investment in start-ups, accelerators, and consortia1. All major banks, as well as Visa, MasterCard, and NASDAQ, are working on blockchain. Dynamic startups such as Ethereum, Blockstream, Ripple, DAH, and Abra are likely to make a big impact as the opportunity evolves. One advancement that blockchain enables is a move to triple-entry bookkeeping. Triple-entry bookkeeping is a new development in accounting that will eradicate the need for expensive, time-consuming duplication of processes between two parties during a transaction. The existing accounting process is streamlined. The distributed ledger will do away with the exchange of invoices and receipts, and their manual entry into individual accounts. Instead of entering transactions separately into independent sets of books, they are automatically, reliably, and efficiently updated in a third ledger, shared between both parties. Using digitally signed receipts and cryptographic authentication, the third ledger is inherently approved by and accessible to both parties [7].

Blockchain has applications outside of F\&A and is fast becoming a conduit for implementing an integrated business services model. The enhanced visibility the blockchain offers makes it much easier to oversee even complex supply chains. The ability to track and trace shipments can reduce errors and disputes, while the instant provision of digital letters of credit and bills of landing can revitalize logistics. Automated smart contracts reduce paperwork and delays, and improve cash flows throughout a supply chain. Platforms such as that offered by Skuchain or Hijro bring new levels of transparency and security to supply chains. They provide optimal planning and agility for downstream buyers and working capital relief for upstream suppliers. One area in which blockchain is clearly unifying disparate parts of businesses is in security and field operations. Every asset, device, and worker can be issued a blockchain-backed digital identity as part of an enterprise-wide trust network. This allows instant monitoring and authentication for every worker across every asset in the business, reducing the likelihood of security failure by removing any single point-of-failure [7].

As with all other business decisions, the decision to apply Blockchain technology involves a cost factor. Since it is designed to facilitate interactions between organizations, 
someone will have to act as the "host", at least for permissioned Blockchains. Beyond licensing the technology itself, the primary costs are infrastructure (hosting) and the energy required for computing. Many transactions are needed to validate blocks, and Blockchain is unlikely to be less expensive than slower, more traditional alternatives. When individual consumers benefit from the implementation of Blockchain, such as in supply chain traceability applications, B2C companies are expected to assume the cost, but when suppliers are the primary beneficiaries, Blockchain may become a new factor in invoice payment discounting. There is not one blockchain, but rather a number of companies and programmers experimenting with applications for distributed ledger technology. This has resulted in multiple competing standards with limited interoperability, diminishing the potential value to be gained by creating visibility all the way back through the supply chain and making it difficult to operate within individual industries.

There is an effort underway involving the International Organization for Standardization (ISO/TC 307) that would establish standards for terminology and concepts related to blockchain and distributed ledger technologies, but with the speed of development, companies may be faced with the need to make decisions before such a standard is accepted and put into practice. In a fully distributed blockchain, there is no need for a central administrator because the block-level cryptography makes the data stored in them immutable.

Cryptocurrency blockchains are always on the lookout for something they call a " $51 \%$ attack." Under a 51\% attack, a group of "miners" (programmers) would have a controlling interest in the blockchain, allowing them authority over all of the blocks seeking authorization. 13 in July of 2014, mining group ghash.io briefly went above the $50 \%$ point in the Bitcoin blockchain. The group voluntarily reduced its share of the network and put a plan in place to prevent them from going above $40 \%$ in the future. In a permissioned blockchain (such as we expect to see implemented in a B2B scenario) there might not be such altruism. If something goes wrong, there are no regulations to govern blockchain-based transactions [8].

A growing number of companies have expressed their will to enter the blockchain arena. But after some number of years in which their focus was mainly on the benefits of blockchain in various areas, in terms of speed, costs, streamline operations and increased efficiency, their attention is now turned to the various challenges and bottlenecks that are preventing widespread adoption. First of all, there is a reputation challenge. Blockchain is still very much connected to the crypto world in the mind of many. And that is seen as a world of bad actors, hackers, frauds and speculators. But more important are the technical ones such as immaturity (still slow and cumbersome), lack of scalability, lack of interoperability, stand-alone projects, difficult integration with legacy systems, complexity and lack of blockchain talent. What to think about the organizational challenges at corporates like lack of good governance, lack of awareness and understanding, lack of user experience and education, the attitude of incumbents, or the security and privacy challenges, including lack of regulation. And finally, but not unimportant other challenges such as culture, energy consumption/environmental cost [9].

Tracking down contaminated food has historically been a messy affair, sometimes taking retailers and food companies weeks to learn exactly what products they need to pull from store shelves. "Imagine if we could pinpoint with certainty within minutes, not 
days," mused Frank Yiannas, one of Walmart's top food safety executives, in a recent video published by the retailer. Thanks to technology originally designed to monitor cryptocurrency, Yiannas' vision is closer to becoming a reality-something that could put a significant dent in the number of foodborne illnesses that occur every year. It's part of a new program in which IBM is partnering with Walmart, Nestlé, Dole, Tyson Foods, Kroger, and others, to use blockchain technology to track food throughout the complex global supply chain. Massive companies putting pressure on suppliers to use blockchain will migrate a huge swathe of the food world into one organized system. That is no small feat when farmers, processors, distributors, and retailers are currently use different types of documentation to track their products - some of them still on paper. Under the new system, if a consumer falls ill from E.coli traced to a batch of lettuce, a food-safety investigator could conceivably scan a barcode on the packaging to quickly learn where it came from and where other lettuce from the same batch went. Retailers will be able to quickly remove contaminated products from shelves, thus stopping the spread of illnesses. Walmart has been using a pilot version of the technology, showing how blockchain can be expanded beyond the financial, health care, and natural resources sectors to be applied to the foods that consumers interact with every single day. Coupled with companies' efforts to stop food-borne illnesses early on, this could signal a major moment in how humans keep the food system in check [10].

Large companies are always battling with their legacy applications, because these can be anchors that drag them when new technologies arrive. Even when you thought corporate IT was safe with modern software environments that make use of modular cloudbased capabilities, container-based-technology to facilitate operations deployments, or continuous delivery with agile and rapid developments practices, the blockchain is yet another "modern technology" that will need to be absorbed and integrated into the technology toolset of any software development teams.

In addition to internal applications and use cases, there will be a number of new opportunities for creating shared blockchain services either at the vertical level (e.g., a particular financial services application), or at the horizontal level (e.g., a generic records verification service). Companies will need to decide what implementation approaches to choose, based on their own competencies and choice of external partnerships. You should not just see the Blockchain as a problem-solving technology. Rather, it is a technology that lets you innovate and target new opportunities [11].

Blockchain is often seen as a system of record that ensures data integrity through ledgers that are immutable distributed systems that cannot be deleted or updated. In other words, Blockchain ensures the integrity of the data. Whether it is the public Blockchain like Ethereum or a Hyperledger based private Blockchain system, they can easily work as a system of records. Even though it may fit well for certain uses, there are some limitations to using Blockchain as a system of records:

- A traditional database has Create, Read, Update and Delete options for managing data, often called CRUD. With Blockchain, only Create and Read functions are allowed. Update is done by creating a new record and Delete is not an option

- People are already using a system of records that is more versatile and data is a big issue in moving to a new system of records. An ideal scenario would be to use the existing system of records, with extra integrity provided by another layer 
- Even though using Blockchain as a system of records builds integrity into the system, it comes with a performance hit, extra cost, scale concerns and other very real issues

A better approach is to use your existing business system and use Blockchain as a "system of integrity" for the data. With this approach, the limitations of blockchain as a system of record is overcome, while still ensuring the integrity of your data by taking advantage of the immutability of blockchain. Salesforce, database services in the cloud, cloud storage solutions like AWS S3, on-premises databases or even SaaS offerings can act as a system of record while blockchain can serve as the system of integrity. Traditional cryptographic fingerprinting techniques like Hashing or Checksums when used with Blockchain as the storage for these digital fingerprints, can serve as a battle tested way to ensure data integrity. In other words, blockchain is an additive to your existing system of record, not a replacement. When you create data, it is easy to add a digital fingerprint to the data that can be a marker to ensure the integrity of that data. This fingerprint is then stored in a safe place and can be called upon anytime to verify the original data integrity. Since blockchain is immutable and decentralized, it can safely store the fingerprints and act as a military grade data integrity platform. By using blockchain as the integrity layer, organizations can continue using their existing systems of record while also staying compliant with any data integrity requirements [12].

\section{Data Collection Method}

The purposes of this research are to (1) explore the current blockchain use cases in Shared Services (2) understand the value created by blockchain in Supply Chain Management and (3) study the tactical challenges in adopting a blockchain strategy in Shared Services. In addition to literature review, we conducted in-depth Interviews and/or sent questionnaires to Shared Services Leaders and experts.

\subsection{In-Depth Interviews/Questionnaires}

In addition to literature review, in-depth interviews were conducted with 13 executives and experts from 12 industries. In cases where the interviews couldn't be arranged, the questions were emailed to the participants. The demographics of the participants are shown in Table 1 below.

The purpose of the in-depth interviews/questionnaires was to seek answers to the following 14 questions:

1. What is your understanding about Blockchain technology?

2. Has your company adopted Blockchain technology in any functional area? If yes, which ones?

3. Some experts believe that Blockchain technology will be bigger than Robotic Process Automation for the Shared Services industry. What is your opinion?

4. In your opinion, what does this game-changing Blockchain technology mean for Shared Services? 
5. What are the key characteristics that facilitate adoption of Blockchain technology in Shared Services?

6. What are the expected benefits of adopting Blockchain technology in Shared Services?

7. What are the potential areas in Finance Shared Services for adopting Blockchain technology in order to improve data integrity?

8. What are the potential areas in HR Shared Services for adopting Blockchain technology in order to streamline HR processes?

9. What are the potential areas in Procurement \& Supply Chain Shared Services for adopting Blockchain technology in order to improve validation, authentication, traceability and transparency?

10. What are the potential opportunities in other areas of Shared Services for adopting Blockchain technology in order to improve visibility of business processes?

11. Are you familiar with the benefits achieved by Walmart with adoption of Blockchain technology? Can your company realize similar benefits?

12. What are the challenges you foresee or have encountered with the adoption of Blockchain technology in Shared Services?

13. Our research indicates that Blockchain technology is being used to replace paper trails, improve validation and authentication, and increase visibility and transparency. What is your opinion?

14. As part of our research, we will be providing business cases for adopting Blockchain technology. Are your executives open to adopting Blockchain technology in your company?

The answers to the questions received from 17 respondents ( $35 \%$ response rate) are summarized in Sect. 4 and analyzed to develop insights about the adoption of Blockchain technology in various areas of Shared Services.

Table 1. In-depth interviews/questionnaires: demographics of participants

\begin{tabular}{l|l|l|l|l}
\hline S. No. & Industry & Location & Position & No. of participants \\
\hline 1 & Health Services & Canada & N/A & 1 \\
\hline 2 & Nonprofit Organization & Canada & $\begin{array}{l}\text { Senior Director - People } \\
\text { Services }\end{array}$ & 1 \\
\hline 3 & Banking & Canada & Various & 5 \\
\hline 4 & Pharmaceuticals & UK & FSSC Manager & 1 \\
\hline 5 & IT & China & Various & 3 \\
\hline 6 & Oil \& Gas & China & Various & 3 \\
\hline 7 & $\begin{array}{l}\text { IT \& Management } \\
\text { Consulting }\end{array}$ & China & Various & 3 \\
\hline 8 & IT & USA & Co-founder/CEO & 1
\end{tabular}


Table 1. (continued)

\begin{tabular}{|c|c|c|c|c|}
\hline S. No. & Industry & Location & Position & No. of participants \\
\hline 9 & Banking & Canada & N/A & 1 \\
\hline 10 & Electricity Generation & Canada & Various & 3 \\
\hline 11 & Telecommunications & Canada & Various & 2 \\
\hline 12 & Insurance & Canada & N/A & 1 \\
\hline 13 & Broadcasting & Canada & Various & 3 \\
\hline 14 & Transportation/Delivery & Canada & $\begin{array}{l}\text { Group Leader - Delivery } \\
\text { Management }\end{array}$ & 1 \\
\hline 15 & Health Care Provider & Canada & Various & 2 \\
\hline 16 & Railways & Canada & $\begin{array}{l}\text { Director - Network } \\
\text { Services }\end{array}$ & 1 \\
\hline 17 & Publishing & Canada & Director - SS & 1 \\
\hline 18 & Consumer Retail & Canada & Assistant Treasurer & 1 \\
\hline 19 & Financial Institution & USA & N/A & 1 \\
\hline 20 & Federal Government & Canada & N/A & 1 \\
\hline 21 & $\begin{array}{l}\text { Audit \& Consulting } \\
\text { Services }\end{array}$ & Canada & N/A & 1 \\
\hline 22 & $\begin{array}{l}\text { Data Processing \& } \\
\text { Consulting }\end{array}$ & Canada & Global BPO Director & 1 \\
\hline 23 & Supermarket Chain & Canada & N/A & 1 \\
\hline 24 & Wholesale Bakery & Canada & N/A & 1 \\
\hline 25 & Energy & USA & $\begin{array}{l}\text { SVP - Global Human } \\
\text { Resources }\end{array}$ & 1 \\
\hline 26 & Management Consulting & USA & Managing Director & 1 \\
\hline 27 & Construction & Canada & Various & 2 \\
\hline 28 & Asset Management & Canada & $\mathrm{CFO}$ & 1 \\
\hline 29 & $\begin{array}{l}\text { Consumer Packaged } \\
\text { Meats }\end{array}$ & Canada & $\mathrm{CIO}$ & 1 \\
\hline 30 & Textile Manufacturing & China & Director - Logistics & 1 \\
\hline 31 & $\begin{array}{l}\text { Tax Advisory \& } \\
\text { Consulting }\end{array}$ & USA & $\begin{array}{l}\text { Senior Manager - } \\
\text { Process Automation }\end{array}$ & 1 \\
\hline
\end{tabular}

\section{Results}

Based on the analysis of data collected, key results are summarized as in this section. 


\subsection{Understanding of Blockchain Technology}

Literature review and respondents' answers indicate that there is strong understanding from both a business use case and technology perspectives. From a technology perspective, Blockchain technology allows developers to build applications that have their code executed on a set of globally distributed computers.

From a business perspective, it allows removal of the requirement of trust between two parties. RPA will help reform the execution of business processes while Blockchain will help provide the support for executing these business process in a secured manner across various technologies and industries. Blockchain will have a bigger user group.

\subsection{Application of Blockchain Technology to Shared Services}

There are many potential applications across Shared Services where Blockchain could provide maximum benefit to the corporate Shared Services organization. Everything from immutable records, timestamps, cost, labor and time reductions to automation are all possible with a Blockchain solution. What is already happening right now is that plenty of organizations are testing Blockchain in their internal operations, often by commissioning Centers of Expertise. In fact, intra-company transactions offer plenty of pain points that present a perfect opportunity for Blockchain. Any place where there is pause for validation or authentication is where Blockchain can automate that need.

Any transaction where 'trust' might be an issue is an opportunity for Blockchain. One example is cross-border payments within an enterprise, which suffer all the same hurdles and loops "as if you are paying a third-party". And whether these transactions are large or small, they are plentiful enough that performance improvement can be achieved. While the value of Blockchain through internal application via Shared Services can be demonstrated, the true wins will accrue in leveraging Blockchain across external transactions, with third parties.

Blockchain technology allows seamless business-to-business transactions based on pre-agreed and confirmed data nodes, through a network. This capability of Blockchain technology empowers businesses, Shared Services in particular, to process decentralized transactions at a higher speed and lower cost essentially by automating the requirement for authentication or validation. Blockchain technology can help share more efficient connections between suppliers, customers, enterprise users and shared service centers. Through the identification of all parties on the authenticity of electronic data, combined with the automatic analysis of data flow, the automation efficiency of Shared Services business processing can be accelerated.

\subsection{Potential Areas for Adopting Blockchain Technology in Shared Services}

Based on our research, the following Table 2 shows potential areas for adopting Blockchain technology in Shared Services:

\subsection{Key Characteristics that Facilitate Adoption of Blockchain Technology}

Key characteristics that facilitate adoption of Blockchain technology include Transparency, Immutability, Audit Trail, Consensus, Finality and Multiple Nodes. 
Table 2. Potential areas for adopting Blockchain in shared services.

\begin{tabular}{|c|c|c|}
\hline $\begin{array}{l}\text { Procurement and supply chain } \\
\text { management }\end{array}$ & Human resources & Accounting \\
\hline $\begin{array}{l}\text { Ordering, Procurement, } \\
\text { Invoicing, Acknowledgement } \\
\text { Logistics Visualization: Track } \\
\text { and Trace Shipments } \\
\text { Letters of Credit } \\
\text { Bills of Lading } \\
\text { Custom Clearance } \\
\text { Smart Procurement Contracts } \\
\text { Stolen Goods/Counterfeit } \\
\text { Products } \\
\text { Inventory Replenishment } \\
\text { Products Return Management } \\
\text { Warranties Management }\end{array}$ & $\begin{array}{l}\text { Employee Identification } \\
\text { Recruitment Process } \\
\text { Access to Academic } \\
\text { Credentials and Certifications } \\
\text { Payroll Process for } \\
\text { International Employees } \\
\text { Benefits Administration } \\
\text { Employment Contracts } \\
\text { Grievance Management } \\
\text { Storage of Current and Past } \\
\text { Performance Reviews }\end{array}$ & $\begin{array}{l}\text { Bookkeeping Entries } \\
\text { Exchange of Invoices and } \\
\text { Receipts } \\
\text { Approval of Documents } \\
\text { Payment Automation } \\
\text { Credit Control } \\
\text { Auditing Process }\end{array}$ \\
\hline
\end{tabular}

Through the non-tampering of the Blockchain technology, the efficiency of Shared Services business processing can be accelerated. Other characteristics include improving business traceability, reducing business audit workload and reducing Shared Services audit risks by recording physical information.

Using Blockchain technology as a way of removing the reliance on trust between two parties (whether that's in sharing data between both parties or executing a certain business process that's built using code).

\subsection{Expected Benefits of Adopting Blockchain in Shared Services}

Any process requires authentication in order to proceed. Traditionally, this has taken the form of a centralized check effectively, a ledger that acts as a clear authority. Blockchain offers this ledger, but because there are so many partners it is distributed across, and validated by, all of them. Even better, no single party can manipulate or change it. So, this eliminates or limits the need for a central authority.

The expected benefits of adopting Blockchain technology in Shared Services are to improve the efficiency of business processing, improve the authenticity of business, reduce the risk of Shared audit, reduce the transmission of original parts, reduce the operating cost, and reduce repeated data entry. Other benefits include lower cost of executing shared software code without having to verify that it was done correctly.

\subsection{Challenges with the Adoption of Blockchain Technology in Shared Services}

Despite the obvious benefits, real implementations are still few. One problem is actually forming the business network and getting the relevant counterparts together. The challenge is that everyone has to sign up to a common contract and share data in the hyper ledger in order for it to work, so it requires industries to meet and agree application 
between themselves. The other challenge is to coordinate internal system transformation, supplier system transformation, determine what needs to be stored in the Blockchain, and whether to use the external chain or the internal chain. It is necessary to deal with the trust relationship between the company's self-built blockchain and the public network alliance, and there is no set of recognized authority alliance in the Internet at present.

Some of the other challenges include difficulty in defining and aligning standards between different parties, lack of talent, high implementation cost and complexity of future maintenance work. Education is also a challenge as lots of people don't fully understand Blockchain technology. Overinflating the value proposition, too much hype has gone into Blockchain technology that has not materialized or is flat out false. Many enterprises are interested in exploring Blockchain technology but not interested in deploying it fully within the next few years.

\section{Conclusions and Future Research}

\subsection{Conclusions}

Our research conducted indicates that Blockchain technology is being used within Shared Services to replace paper trails, improve validation and authentication and increase visibility and transparency. Blockchain creates a ledger of all transactions in a given process. Each ledger entry is autonomous so can provide insights into cashflow and add extra security. Virtually incorruptible due to the fact that the data contained within a ledger entry cannot be copied, Blockchain can track any item of value, so is particularly useful in shipping as well as online retail, making it a powerful tool for business units with the vision to utilize this technology.

The process of taking any piece of digital data of any size and chopping, mincing and mixing it until it is an unrecognizable string of digits and characters is called hashing. Hashing is one of the core concepts needed to understand blockchain. The other three are Public/Private key cryptography, decentralization and then the blockchain data structure itself. Hashing makes it easy to confirm the authenticity of data when comparing two or more versions. An extra cent in a ledger will be obvious instantly. A missing comma in a contract will make itself immediately apparent. Public-private key encryption, within the context of Blockchain, is what allows to keep track of virtual identities and signatures. A private key allows the owner to encrypt data. A public key allows everybody else to decrypt the data. A private key functions as a digital signature.

\subsection{Future Research}

This research paper introduces the potential for adopting blockchain in Shared Services. It offers the value proposition of introducing blockchain in various functions within Shared Services. The findings and analysis presented here will assist more companies to develop an over-arching strategy for adopting the blockchain technology. Future research is required in the areas of technology service providers and availability of implementation tools. Specifically, future research should focus on answering the following questions: 
1. Which service provider organizations are providing services for digital transformation of Shared Services using blockchain and what investments will create meaningful results for them?

2. What service automation tools e.g. cognitive automation tools are available for automating Knowledge-Based Shared Services?

3. What industries are adopting the service automation strategy to automate their Expertise Services and what implementation approaches have proven to be effective?

4. What are the use cases and the associated challenges, risks and opportunities and how blockchain development and deployment can be managed?

\section{References}

1. Lewis-Kraus, G.: Inside the Crypto World's Biggest Scandal. In: Business, p. 41 (2018)

2. Hodge, B.: Blockchain is a Gamechanger. In: SSON, p. 7 (2018)

3. Andersen, N.: Blockchain Technology: A Game Changer in Accounting. In: Deloitte, p. 5 (2018)

4. Psaila, S.: Blockchain: A Gamechanger for Audit Processes. In: Deloitte (2018)

5. Perroser, R.: Are There Use Cases in Shared services for Blockchain. In: IQPC: Blockchain in Shared Services, p. 7, 18 March 2018

6. Huillet, M.: Accenture, Generali Launch Blockchain Solution for Employee Benefits. In: Cointelegraph, p. 1 (2019)

7. Gopal, V.: Using Emerging Technologies to Build NextGen Shared Services. In: Tata Consulting Services, p. 9 (2018)

8. Biju Mohan, B.K.V.N.: Blockchain in the Real World. In: Smart by GEP, p. 10 (2018)

9. De Meijer, C.R.W.: Remaining challenges of blockchain adoption and possible solutions. In: MIFSA (2020)

10. Purdy, C.: Supermarkets are Now Using Blockchain to Keep Food Fresh. In: Quartz, p. 2, 24 August 2017

11. Mougayar, W.: The Business Blockchain. Wiley, Hoboken (2018)

12. Chainkit: Continuous Data Integrity Using Blockchain (2019). [Online] 